\title{
Association between Electronic Medical Record Implementation of Default Opioid Prescription Quantities and Prescribing Behavior in Two Emergency Departments
}

\author{
M. Kit Delgado, MD, MS, FACEP', 2,3,4, Frances S. Shofer, PhD ${ }^{7}$, Mitesh S. Patel, MD, MBA, MS $35^{3,5}$, \\ Scott Halpern, MD, PhD ${ }^{2,3,5,6}$, Christopher Edwards, $\mathrm{MD}^{7}$, Zachary F. Meisel, MD, MSHP ${ }^{1,4}$, and \\ Jeanmarie Perrone, $M D^{7}$
}

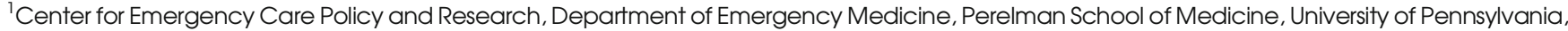
Philadelphia, PA, USA; ${ }^{2}$ Department of Biostatistics, Epidemiology, and Informatics, Perelman School of Medicine, University of Pennsylvania, Philadelphia, PA, USA; ${ }^{3}$ Center for Health Incentives and Behavioral Economics, Perelman School of Medicine, University of Pennsylvania, Philadelphia, PA, USA; ${ }^{4}$ Penn Injury Science Center, University of Pennsylvania, Philadelphia, PA, USA; ${ }^{5}$ Department of Medicine, Perelman School of Medicine, University of Pennsylvania, Philadelphia, PA, USA; ${ }^{6}$ Department of Medical Ethics and Health Policy, Perelman School of Medicine, University of Pennsylvania, Philadelphia, PA, USA.
\end{abstract}

KEY WORDS: opioid prescribing; defaults; EMR.

J Gen Intern Med 33(4):409-11

DOI: $10.1007 / \mathrm{s} 11606-017-4286-5$

(c) Society of General Internal Medicine 2018

\section{INTRODUCTION}

Larger quantities of opioid tablets for initial prescriptions are associated with transition to continued use. ${ }^{1}$ Default options, or conditions that are set in place unless an alternative is actively chosen, have been shown to influence behavior in many contexts, including increasing the rates of prescribing generic versus brand-name drugs to over $98 \%$ in primary care. ${ }^{2,3}$ Leveraging default options in electronic medical record (EMR) prescribing orders thus represents a promising approach to guide clinicians towards prescribing smaller quantities of opioid tablets, thus reducing continued use, misuse, and diversion.

In 2015, the emergency departments (EDs) of the Hospital of the University of Pennsylvania (HUP, annual volume 68,000) and Penn Presbyterian Medical Center (PMC, annual volume 41,000) adopted a new EMR (Epic, Verona, WI) to replace a homegrown EMR (EMTRAC). EMTRAC required the clinician to enter the number of tablets for opioid
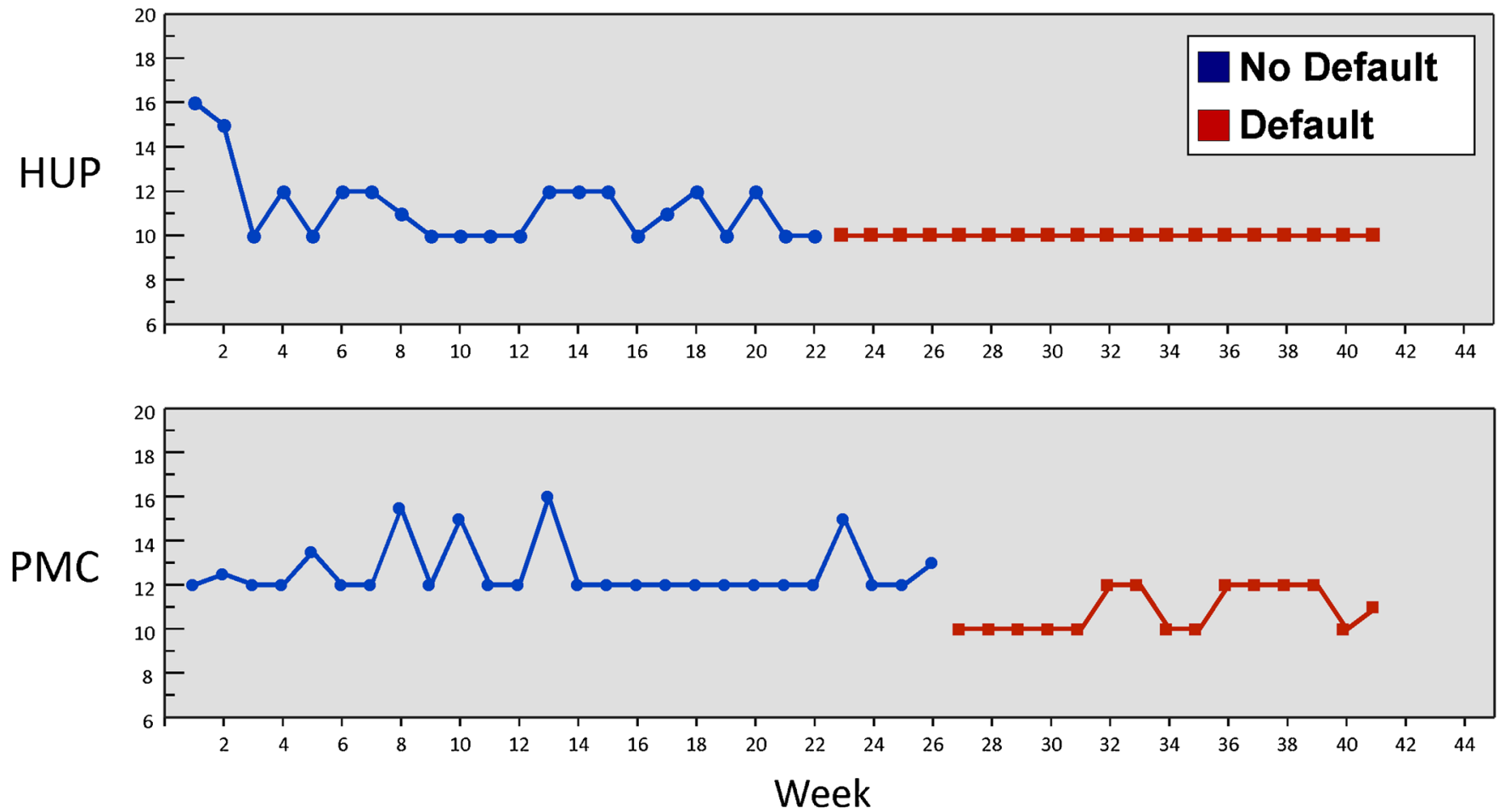

Figure 1 Median number of oxycodone w/ acetaminophen 5-325 mg tablets dispensed per week before and after implementation of emergency department electronic medical record discharge order default of $\mathbf{1 0}$ tablets vs. no default. 
prescriptions. Since the implementation of the new EMR, when a clinician types in an ED discharge opioid prescription order, a preference list appears with a default quantity of 10 tablets displayed first. The clinician can "opt out" by selecting a quantity of 20 tablets, which is displayed second, by modifying either of these orders, or by clicking on "Database Lookup," where a new health system default of 28 tabs is displayed, as well as manual entry options. We evaluated the effect on prescribing behavior associated with the implementation of an EMR opioid default supply quantity for our most common opioid discharge prescription in two EDs.

\section{METHODS}

Using EMR data from October 1, 2014, to June 29, 2015, we compared weekly prescribing patterns in discharge quantities supplied for oxycodone $5 \mathrm{mg} /$ acetaminophen $325 \mathrm{mg}$ (Oxy/ APAP) between the pre-intervention period (weeks 1-22 for HUP and 1-26 for PMC) and the post-intervention period (weeks 23-41 for HUP and 27-41 for PMC). Using a staggered roll-out, two time periods were constructed: (1) 4 weeks prior to HUP ED default implementation (weeks 19-22), and (2) 4 weeks after HUP ED default implementation/PMC no change (weeks 23-26). We used two-way analysis of variance to test for differences in the mean and median number of tablets supplied per week and chi-square tests to determine differences in proportions of prescription quantities.

\section{RESULTS}

During the 41-week period, 3264 prescriptions for Oxy/APAP were written across the two EDs. After the default implementation, there was no change in the mean number of Oxy/APAP tablets prescribed per week across the two EDs $(p=0.42)$, but the median number decreased by a small amount, from 11.3 to $10(p=0.004)$ in the HUP ED and 12.6 to $10.9(p<0.001)$ in the PMC ED (Fig. 1). However, there was a marked increase across the two EDs in the proportion of prescriptions written for 10

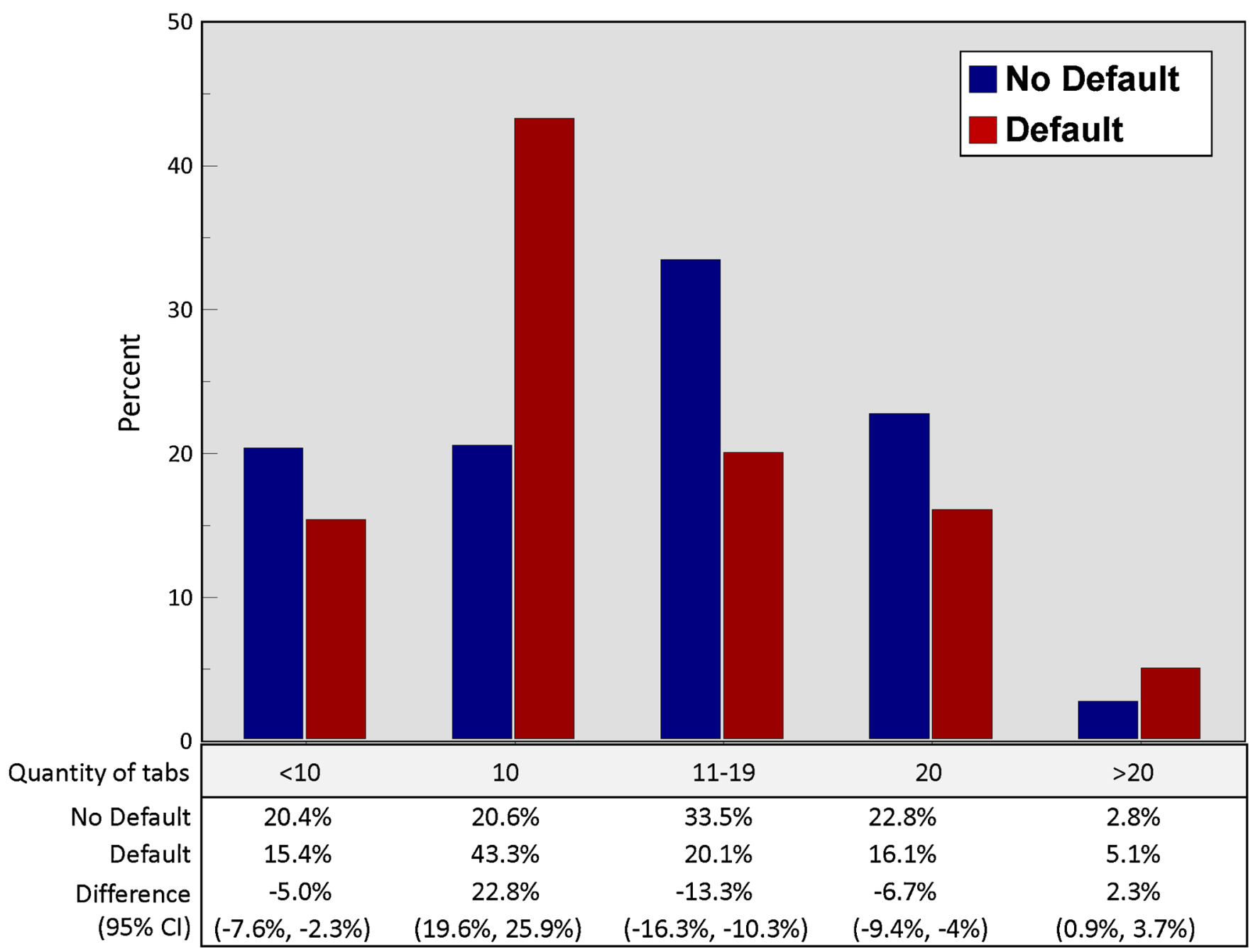

Figure 2 Quantity of oxycodone w/ acetaminophen 5-325 mg tablets dispensed before and after implementation of emergency department electronic medical record discharge order default of 10 tablets vs. no default. The clinician could "opt out" by selecting a quantity of 20 tablets, which was displayed second, by modifying either of these orders, or by clicking on "Database Lookup," where a health system default of 28 tabs was displayed, as well as manual entry options. 
tablets (from $20.6 \%$ to $43.3 \%$, an increase of $22.8 \%$; Fig. 2), and this increase was even higher when comparing the 4 weeks after implementation in the HUP ED relative to the control period in the PMC ED (27.8\% [95\% CI 17.4-37.5\%], $p<$ 0.001 ). The proportion of prescriptions written for 20 tablets, the second listed option in the preference list, actually decreased by $6.7 \%$, as did prescriptions for $11-$ 19 tablets (13.3\%) and $<10$ tablets (5\%). Finally, there was a small increase in the proportion of prescriptions written for $>20$ tabs $(2.3 \%), 50.0 \%$ of which were for the system-wide default of 28 tablets, compared to $4 \%$ in the pre-intervention period $(p<000.1)$.

\section{DISCUSSION}

We found that implementation of an EMR default of 10 tablets versus no default was associated with a strong increase in the proportion of prescriptions written for 10 tablets. However, given that baseline quantities prescribed were already low and were consistent with ED guidelines ${ }^{4}$ there was no clinically significant reduction in the overall quantity supplied. A potential unintended consequence of this intervention was a small reduction in prescriptions written for less than the default option of 10 tablets. This suggests that future efforts to set default quantities should start with the lowest baseline prescription. One other study on ED defaults found that the change from a 15-tablet default to no default resulted in an increase in the quantity supplied. ${ }^{5}$ In conclusion, deploying EMR defaults that include lower-thanbaseline default opioid quantities for acute pain is potentially a widely scalable approach for changing prescribing behavior, while still preserving clinician autonomy.

Acknowledgments: Research reported in this manuscript was supported by the National Institute on Drug Abuse and the National Institute of Child Health and Human Development of the National Institutes of Health under award numbers P30DA04050O (MKD, ZM, JP) K23HD090272001 (MKD). The content is solely the responsibility of the authors and does not necessarily represent the official views of the National Institutes of Health.

Corresponding Author: M. Kit Delgado, MD, MS, FACEP; Center for Health Incentives and Behavioral Economics, Perelman School of Medicine University of Pennsylvania, Philadelphia, PA, USA (e-mail: kit.delgado@uphs.upenn.edu).

\section{Compliance with Ethical Standards:}

Conflict of Interest: Dr. Delgado reports receiving an honorarium for participating in an Expert Roundtable on Opioid Prescribing convened by United Health Group. Dr. Patel is the founder/owner of Catalyst Health, a technology and behavioral change consulting firm, and is a member of the advisory boards of Healthmine, Inc. and Life.io. All other authors declare that they have no conflict of interest.

\section{REFERENCES}

1. Shah A, Hayes CJ, Martin BC. Characteristics of initial prescription episodes and likelihood of long-term opioid use-United States, 20062015. MMWR Morb Mortal Wkly Rep. 2017;66:265-69. https://doi.org/ 10.15585/mmwr.mm6610a1.

2. Halpern SD, Ubel PA, Asch DA. Harnessing the power of default options to improve health care. N Engl J Med 2007;357(13):1340.

3. Patel MS, Day SC, Halpern SD, Hanson WC, Martinez JR, Honeywell S, Volpp KG. Generic medication prescription rates after health system-wide redesign of default options within the electronic health record. JAMA Intern Med. 2016;176(6):847-8.

4. Weiner SG, Baker O, Poon SJ, Rodgers AF, Garner C, Nelson LS, Schuur J. The effect of opioid prescribing guidelines on prescriptions by emergency physicians in Ohio. Ann Emerg Med. 2017;70(6):799-808.

5. Zwank MD, Kennedy SM, Stuck LH, Gordon BD. Removing default dispense quantity from opioid prescriptions in the electronic medical record. Am J Emerg Med. 2017;35(10):1567-69. 